\title{
Implementation of an Electric Motor for Light River Transportation Units
}

\section{Implementación de un Motor Eléctrico para Unidades Fluviales Ligeras de Transporte}

\author{
J. Pancha, V. Rojas, V. Romero, and J. Néjer \\ Escuela Superior Politécnica de Chimborazo, Riobamba, Ecuador
}

VII International Congress of Science, Technology, Entrepreneurship and Innovation (SECTEI 2020)

Corresponding Author:

V. Romero

Vicente.romero@espoch.edu.ec

Published: 26 August 2021

Production and Hosting by

Knowledge E

(c) J. Pancha et al. This article is distributed under the terms of the Creative Commons Attribution License, which permits unrestricted use and redistribution provided that the original author and source are credited.

\section{Abstract}

The fluvial transport is the way that allows the development of the communities in post of improve the communication, but its use with combustion engines causes an environmental impact and damage to the ecosystem by emitions, noise pollution or oil spill. In fact, to finding other alternatives to improve operating conditions and reduce environmental impact, the application of electric motors being a new alternative for river transport. In this design, two direct current motors will applied, such as the propulsion of an environmentally friendly light boat coupled with a set of energy accumulators that supply the propulsion system. The applied motors and the type of battery used indicate in the design characteristics. This analysis shows the results obtained in the simulations and designs of the boat in relation to the resistance generated by the displacement in the water and the aerodynamic flow conditions.

Keywords: river propulsion, electric.

\section{Resumen}

El transporte en lugares fluviales es un medio importante que permite el desarrollo de las poblaciones impulsando la comunicación entre comunidades, pero su uso con motores de combustión interna provoca un impacto ambiental y daños al ecosistema por emisiones, contaminación acústica o derrame de derivados del petróleo. Esto conlleva a encontrar otras alternativas para mejorar las condiciones de operación y reducir el impacto ambiental siendo la aplicación de motores eléctricos una nueva alternativa para el transporte fluvial. En este diseño aplica dos motores de corriente continua como propulsión de una embarcación ligera amigable con el medio ambiente acoplado con un conjunto de acumuladores de energía que abastecen al sistema de propulsión. Se indica además en las características de diseño los motores aplicados y del tipo de batería usada. En este análisis se muestran los resultados obtenidos en las simulaciones y diseños de la embarcación en relación a la resistencia generada por el desplazamiento en el agua y además las condiciones del flujo aerodinámico.

Palabras Clave: propulsión fluvial, motor eléctrico, embarcación.

\section{Introducción}

La aplicación de sistemas eléctricos en la propulsión de embarcaciones a gran escala A OPEN ACCESS ha tenido una mayor aplicación en diferentes diseños, convirtiéndose como una forma alternativa al uso de un motor de combustión interna [1]. El diseño de una embarcación 
ligera para el transporte de personas siendo la característica del sistema de propulsión el uso de energía almacenada para abastecer a un motor eléctrico. En el desarrollo de este documento se detallan las características que tiene el sistema de propulsión para este tipo de embarcación ligera como su desempeño en condiciones fluviales que se obtiene con la aplicación de simulación, lo que permite analizar el desempeño de este tipo de embarcaciones ligeras.

\subsection{Emisiones contaminantes}

El cambio climático es una muestra de que se debe cambiar o buscar nuevas formas de transporte en relación a la actual, la generación constante y masiva de emisiones de gases causantes del conocido efecto invernadero siendo el campo del transportación marítima un eje fundamental de estudio en relación a la emisión estos gases contaminantes [2]. Se considera que la contaminación de embarcaciones se clasifican en Bióticos tales como bacterias, algas, hongos, etc.; que son contaminantes que tiene vida y que pueden causar variaciones en el ecosistemas por el desplazamiento de las embarcaciones [2].

Mientras los contaminantes Abióticos son aquellos contaminantes químicos como los hidrocarburos utilizados en motores de combustión interna generando una subdivisión sobre ecosistemas costeros siendo la de más impacto en contaminación química, eutrofización, gases de efecto invernadero [2]. Los océanos se han convertido en un ente receptor de gran cantidad de contaminantes especialmente en lugares de la costa o en lugares fluviales ya que cerca de ésta existe mayor acción por parte de actividades marítimas [3]. Organismos internacionales como el de Protección del Medio Marino con base direccional de la OMI, trabaja por la implementación de técnicas y operación para mejorar la eficiencia energéticas, nuevas tecnologías en propulsión, energías renovables y emisiones de gases de efecto invernadero [4].

\subsection{Motor eléctrico}

La aplicación de un motor eléctrico para lograr la propulsión de una embarcación ligera se ha optado por el uso del motor de corriente continua donde es indispensable contar con un control de velocidad en el cual se aplica un elemento encargado de variar el voltaje de alimentación hacia el motor para lograr tener el cambio en la velocidad de la embarcación e incluso tener el comando en el motor para lograr cambiar el sentido de giro del motor [1]. Además para lograr manejabilidad y acoplar la propulsión a la embarcación es importante a través de un timón cuya gobernación tiene asistencia hidráulica para que la maneabilidad de la propulsión sea más ligera evitando el esfuerzo del timonero. Es importante considerar que la limitación de un sistema propulsado de 
forma alternativa es la generación o acumulación de energía se refleja en la autonomía. Esto limita casi exclusivamente a cascos de desplazamiento en el rango de bajas velocidades, donde la resistencia al avance es mínima [1]. Por lo tanto las velocidad de la embarcación es directamente proporcional a la cantidad de energía que se exista como capacidad disponible. Dichas velocidades se logran alcanzar en relación al límite de navegación de desplazamiento para ciertas embarcaciones ligeras, logrando obtener valores según el Número de Froude (Fn) que alcanzan a $\mathrm{Fn}=0,4$ [5]. Una de las características de una embarcación con una propulsión de un motor eléctrico es la posible falta de la densidad energética del acumulador de engería que puede estar en valores 1-1,4 MJ/kg) en relación con la de un combustible que puede estar entre 43-47 $\mathrm{MJ} / \mathrm{kg}$, una embarcación con una movilidad alternativa tiene un viabilidad e aplicación de transporte [6].

\section{Metodología}

\subsection{Diseño preliminar}

Considerando diseños actuales de embarcaciones para el transporte marítimo ligero se opta por un prototipo que cumpla con los requerimientos para el transporte de personas. El diseño mostrado en la Figura 1, fue utilizado para simular y calcular la resistencia al agua y al aire que va a ejercer el bote, para ello se utilizó el Software Siemens NX 10.

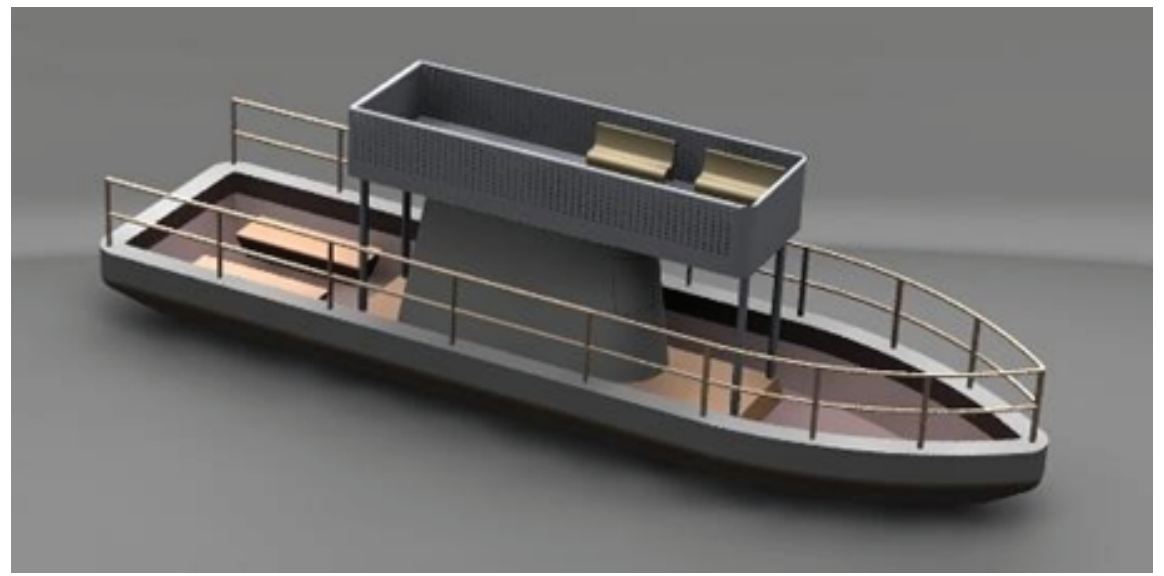

Figure 1

Diseño de la embarcación con propulsión alternativa.

En la Figura 2 muestra la vista frontal del diseño propuesto, considerando que la aplicación de la embarcación es en movilidad fluvial y en comparación a las dimensiones con otros diseños especialmente con propulsión de un motor combustión interna, se busca aprovechar los espacios libres para la ubicación de baterías. 


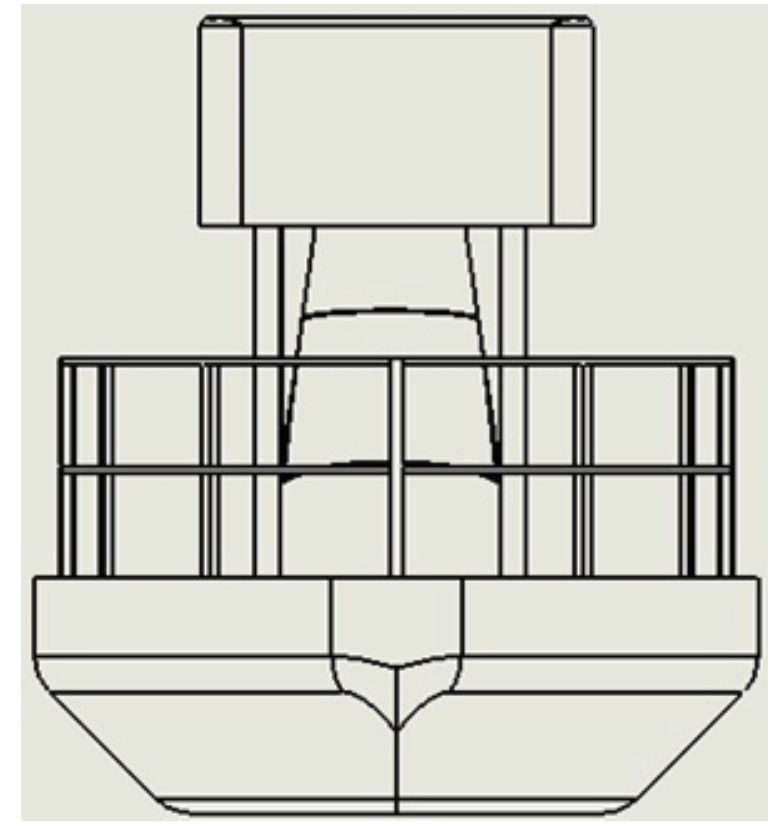

Figure 2

Vista frontal de incidencia.

Mediante una simulación CFD (Control dinámico de fluidos), se considera las siguientes condiciones a una presión máxima que se genera al momento de navegar en las condiciones fluviales y el aire en la parte frontal del diseño, siendo esta la más crítica de la evaluación.

\section{Table 1}

Condiciones de estudio para la resistencia al desplazamiento de la embarcación.

\section{Velocidad Área}

frontal $\left(m^{2}\right)$
Aire

$(\mathrm{km} / \mathrm{hr})$
50
4,18

La Tabla 2 muestra las principales características dimensionales de la Agua 50 3,21 embarcación diseñada para reducir con la mayor capacidad de transporte de objetos y personas cuyo casco se ha estructurado en su fabricación con materiales compuestos debido a que el sistema de propulsión está ubicado homogéneamente en toda la cubierta permitiendo la mejor optimización de su espacio logrando obtener un estructura liviana en relación a los diseños convencionales.

\subsection{Adaptación sistema eléctrico}

El sistema eléctrico de la propulsión de la embarcación ligera diseñada, utiliza dos circuitos de control de 48 voltios de corriente continua, como se ve en el esquema de la Figura 3, como propulsión de le embarcación utiliza una fuente de energía tipo 
Table 2

Características dimensionales de la embarcación.

Eslora total
Manga
Puntual
Calado
Desplazamiento
Pasajeros
Tripulación
Velocidad media
Velocidad máxima
Potencia motor
Corriente continua
Material del casco
Tiempo estimado de carga

$9,65 \mathrm{~m}$
$2,56 \mathrm{~m}$
$2,8 \mathrm{~m}$
$1,2 \mathrm{~m}$
$4,5 \mathrm{t}$
10
2
$55,38 \mathrm{~km} / \mathrm{hr} 7 \mathrm{hr} \sim 30$ nudos
$83 \mathrm{~km} / \mathrm{hr} 2,5 \mathrm{hr} \sim 45$ nudos
$11 \mathrm{KW}$
$48 \mathrm{~V}$
Compuesto
$6 \mathrm{hr} 110 \mathrm{~V} \sim 4,3 \mathrm{hr} 220 \mathrm{~V}$

acumulador que abastece a todos los circuitos de comando y un controlador electrónico para la gestión de cada motor como se observa en la Figura 4, el cual es alimentado mediante un relé de alta capacidad de corriente.

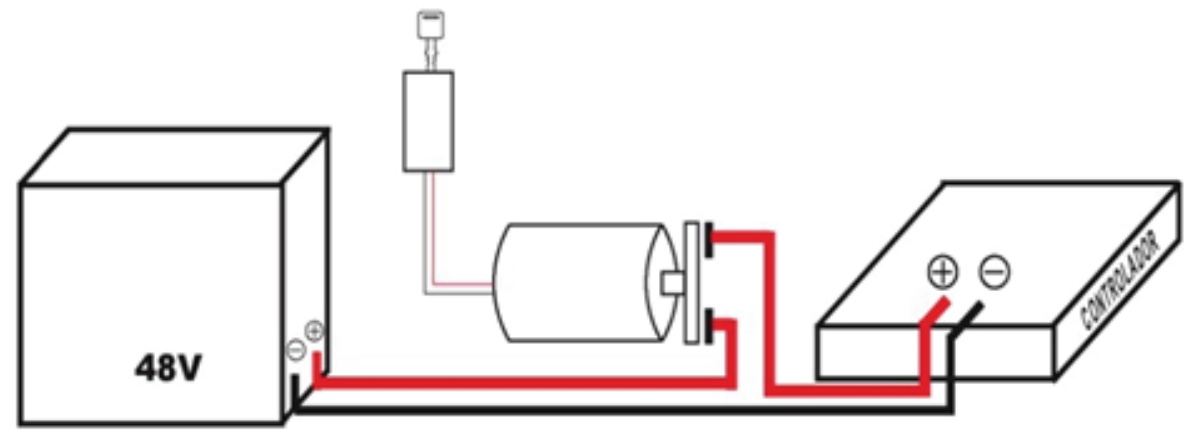

Figure 3

Circuito de control de la propulsión.

\subsection{Batería fosfato de litio Li3PO4}

La batería a utilizar debe abastecer al sistema eléctrico con una tensión salida de 48 voltios de corriente continua y un consumo de 17,37 amperio; respecto al diseño y la aplicación fluvial a la que se requiere llevar esta embarcación es necesario un acumulador de una mayor de una capacidad disponible con un material activo formado por fosfato de litio como se ve en la Figura 5 con las características de 314 voltios de corriente continua y una capacidad disponible de 125 amperios hora. 


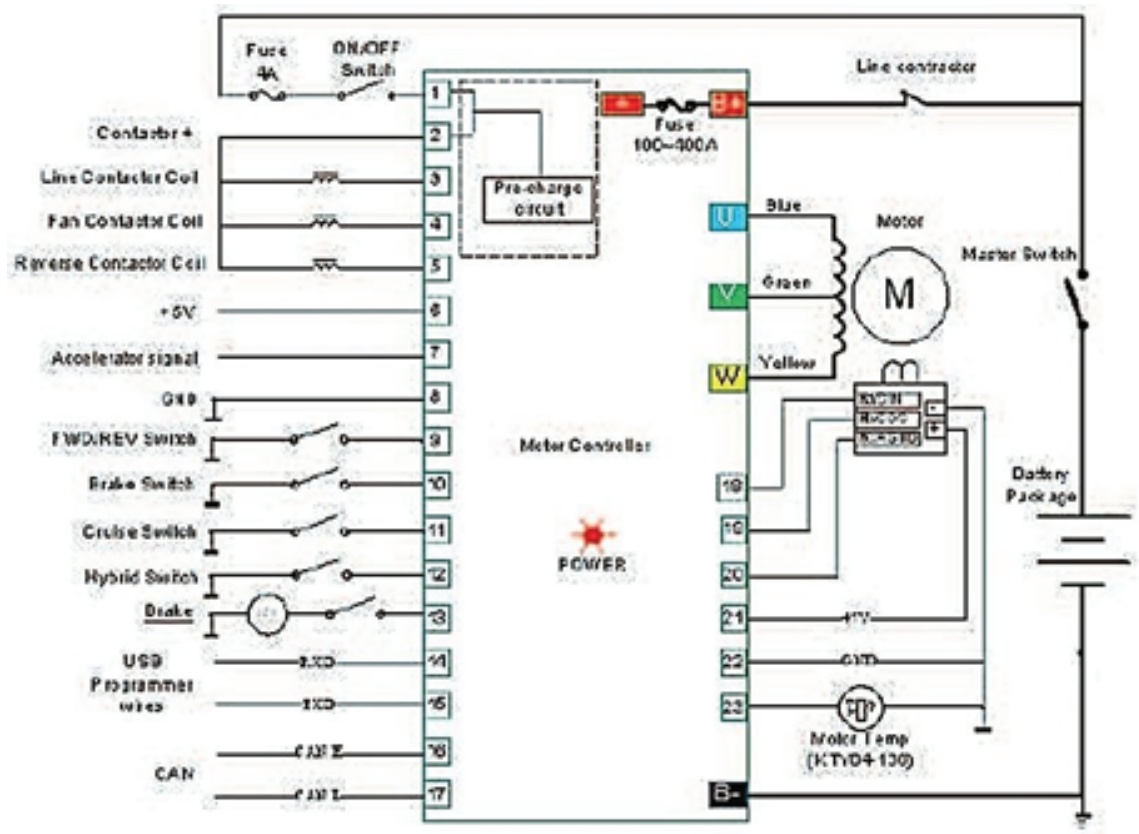

Figure 4

Diagrama de conexión controlador.

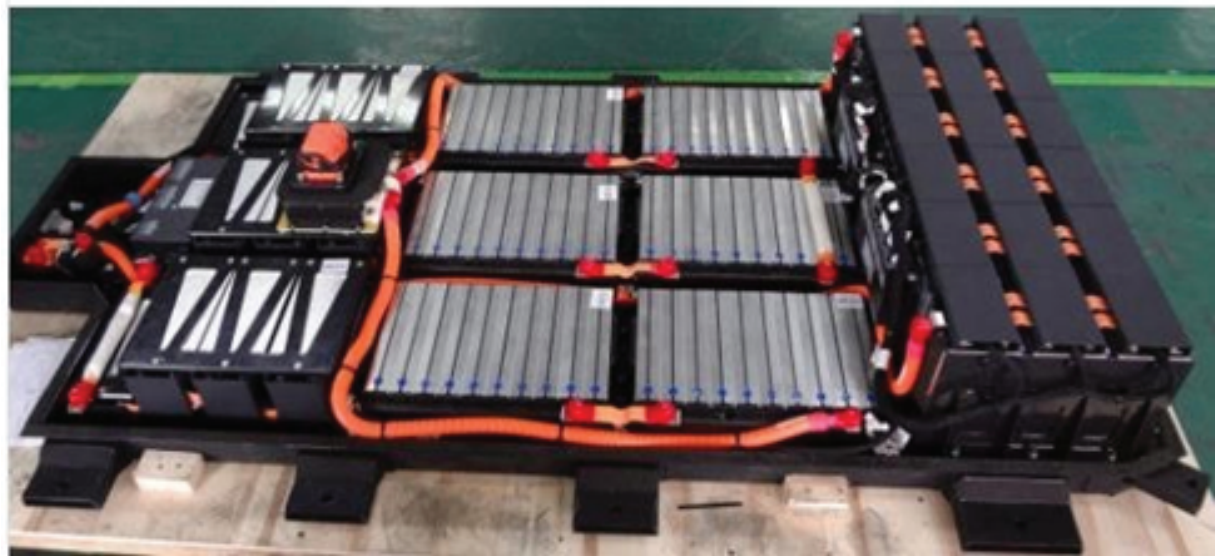

Figure 5

Bacteria Li3PO4.

El acumulador que se usa para abastecer de energía al sistema eléctrico de propulsión como se mencionó anteriormente es de fosfato de litio, las características eléctricas y de diseño se presentan el Tabla 3. Esta generación de baterías tiene aplicación en vehículos. 
Table 3

Características baterías Li3PO4.

Artículo
Voltaje nominal
Gama del voltaje
Capacidad clasificada
Energía total
Tipo de la célula
Poder continuo de la descarga
Temporeros de trabajo. Gama
Tarifa de la auto descarga
Nivel de la protección
Peso

\begin{tabular}{|c|c|}
\hline Datos de la tecnología & Observación \\
\hline \multicolumn{2}{|l|}{$313,2 \mathrm{~V}$} \\
\hline \multicolumn{2}{|l|}{$261 \mathrm{~V} \sim 365,4 \mathrm{~V}$} \\
\hline \multicolumn{2}{|l|}{$100 \mathrm{Ah}$} \\
\hline \multicolumn{2}{|l|}{$31,3 \mathrm{KWh}$} \\
\hline \multicolumn{2}{|l|}{$\mathrm{NCM}$} \\
\hline \multicolumn{2}{|l|}{$30 \mathrm{KW}$} \\
\hline \multicolumn{2}{|l|}{$-10 \sim 45^{\circ} \mathrm{C}$} \\
\hline el $5 \%$ & 30 days, $25^{\circ} \mathrm{C}$, el SOC100\% \\
\hline \multicolumn{2}{|l|}{ IP67 } \\
\hline 330 kg & 5 personas \\
\hline
\end{tabular}

\subsection{Motor}

En el diseño del sistema de propulsión se usa un motor de corriente continua continúa sin escobillas BL-DC, este tipo de motores con potencia de 10 ○ $20 \mathrm{KW}$ son usados en este tipo de embarcaciones. Las características de motor seleccionado se muestran en la Tabla 4.

\section{Table 4}

Caracteristicas motor BLDC.

Especificaciones
Motor
Potencia máxima de entrada
Potencia máxima de salida
Par máximo
Eficiencia
Refrigeración
Peso
Voltaje
Rpm
Diámetro
longitud

Tipo/unidad
BLDC
$11,575 \mathrm{KW}$ a 3304 RPM
$10,012 \mathrm{KW}$ a 3304 RPM
$28,94 \mathrm{Nm}$ a 3304 RPM
$86,5 \%$
Por aire
$17 \mathrm{~kg}$
$48 \mathrm{~V}$
$3000-6000$
$206 \mathrm{~mm}$
$170 \mathrm{~mm}$

\section{Resultados}




\subsection{Resistencia al agua}

Solucionando el sistema propuesto se halla la presión máxima que se va a ejercer en la parte que se sumerge del bote.

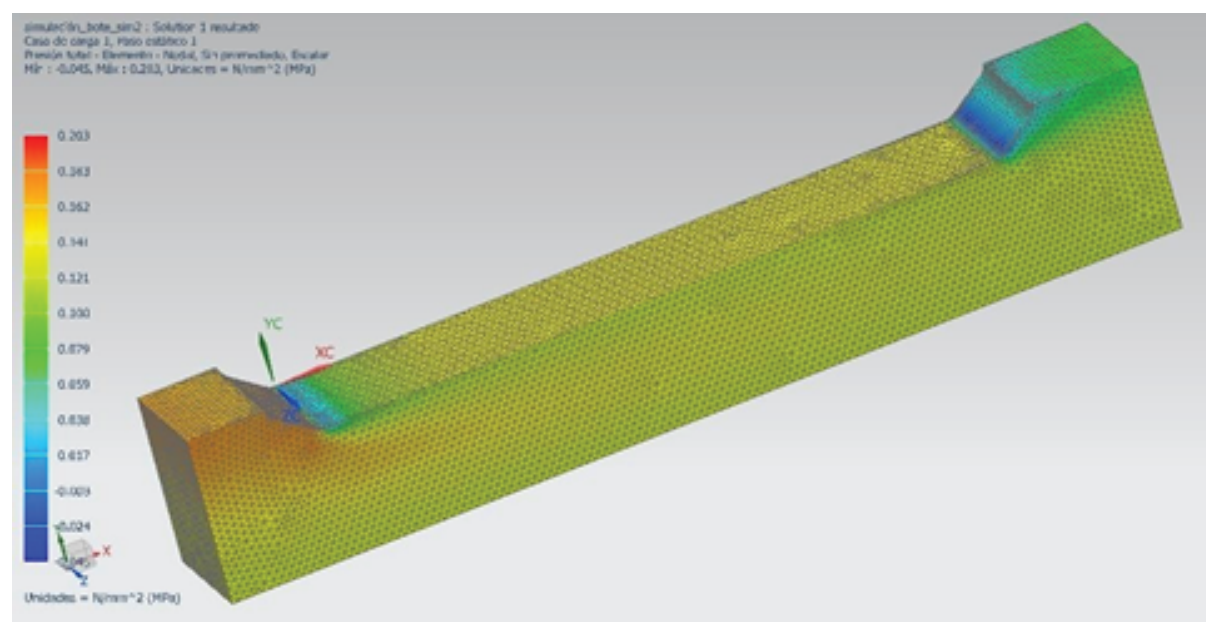

Figure 6

Presión del Agua a 203.01 Pa.

\subsection{Resistencia al aire}

De igual manera y con las condiciones establecidas, se procede a calcular la presión máxima que ejerce el aire en el área frontal del bote sin sumergir.

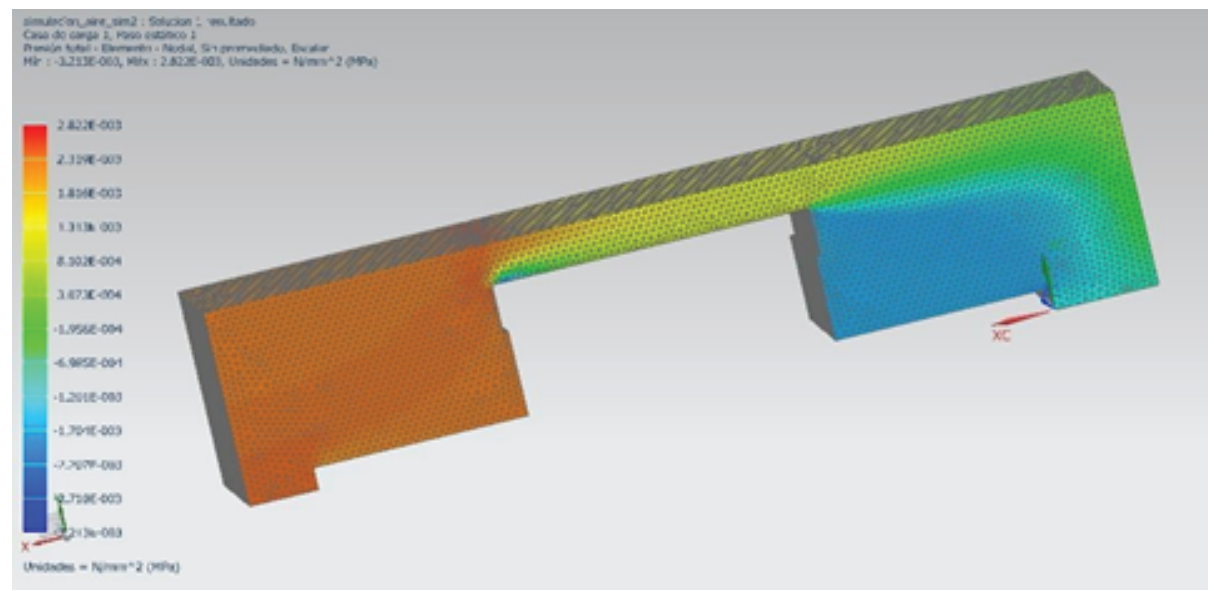

Figure 7

Presión del Aire a 21.9 Pa. 
Con los valores de presión hallados se procede a calcular la fuerza que ejerce el aire y el agua sobre el bote para que se movilice a la velocidad descrita anteriormente.

$$
\begin{gathered}
P_{\text {agua }}=\frac{F_{\text {agua }}}{A}, \\
F=P_{\text {agua }} * A \\
F_{\text {agua }}=203,01 \mathrm{~Pa} * 3,21 \mathrm{~m}^{2} \\
F_{\text {agua }}=651,66 \mathrm{~N} .
\end{gathered}
$$

La fuerza que ejerce el agua sobre el bote es de 651,66 Newtons.

$$
\begin{gathered}
P_{\text {aire }}=\frac{F_{\text {aire }}}{A}, \\
n \\
F_{\text {aire }}=P_{\text {aire }} * A \\
F_{\text {aire }}=21,9 \\
P a * 4,18 m^{2} \\
F_{\text {aire }}=91,54 \mathrm{~N}
\end{gathered}
$$

La fuerza que ejerce el aire sobre el bote es de 91,54 Newtons.

Con estos dos valores podemos calcular la fuerza total que necesita superar el motor eléctrico para poder llevar a cabo el arrastre del prototipo.

$$
\begin{gathered}
F_{\text {Total }}=F_{\text {agua }}+F_{\text {aire }} \\
F_{\text {Total }}: 65,66 \mathrm{~N}+91,54 \mathrm{~N} \\
F_{\text {Total }}: 743,20 \mathrm{~N} .
\end{gathered}
$$

Con este resultado podemos concluir que el motor eléctrico es capaz de arrastrar la panga diseñada.

Formula de velocidad promedio.

$$
v=\frac{d x}{d t}
$$




$$
\begin{gathered}
v \int_{t 0}^{t 1} d t=\int_{x 0}^{x 1} d x \\
v(t 1-t 0)=x 1-x 0 \\
v(6.5-0)=100-0 \\
v=\frac{100 \mathrm{~m}}{6.5 \mathrm{~s}} \\
v=15.385 \mathrm{~m} / \mathrm{s} \\
v=55.38 \mathrm{Km} / \mathrm{h} .
\end{gathered}
$$

\section{Table 5}

Velocidades y aceleración individual.

Velocidad máxima en eficiencia
\begin{tabular}{ll} 
Estimación & $55.38 \mathrm{Km} / \mathrm{hr}$ \\
\hline Tiempo & $6.5 \mathrm{~s}$
\end{tabular}

Velocidad máxima
alcanzada
$83 \mathrm{Km} / \mathrm{hr}$
$20 \mathrm{~s}$

Aceleracion máxima $1.153 \mathrm{~m} / \mathrm{s} 2$

$20 \mathrm{~s}$

Table 6

Autonomia de duración batería.

\section{Recorrido (km) 3640}

Tiempo

$6 \mathrm{hr}$

$7.5 \mathrm{hr}$

Velocidad máxima promedio

(km/hr)

Aparecerán con un texto de una secuencia lógica, y si se dan en tablas, gráficos, etc., no deberán repetirse en él los mismos datos. Se procurará resaltar las observaciones más importantes, describiendo sin interpretar ni hacer juicios de valor, las efectuadas sobre el material y métodos empleados.

Las imágenes, tablas, esquemas o gráficos se situarán preferentemente como se indica en el apartado anterior. En caso de que precisen mayor tamaño para ser visibles, se facilitarán aparte colocando solamente los pies en el lugar deseado.

Las ecuaciones se expresarán dejando líneas de separación entre el texto anterior y el posterior. Si van numeradas, dar el número al final de la línea y cuidar la numeración consecutiva. Si superan la longitud de línea se facilitarán aparte colocando solamente el número en el lugar deseado, tal como se indica en el apartado anterior. 


\section{Conclusiones}

El proyecto de estudio demuestra una mejora para la vialidad de las estructuras de las embarcaciones, ya que permite a más de la reducción de emisiones ambientales y económicas, una ayuda para el camuflaje del desarrollo de la embarcación debido a que este sistema es prácticamente silencioso.

El diseño estructural permite colocar el $80 \%$ del sistema de abastecimiento de energía en la parte superior de la embarcación, permitiendo así en su cubierta tener un mayor espacio de almacenamiento y mejorando la distribución de cargas a lo largo de la misma, estableciendo un mejor comportamiento dinámico y aerodinámico.

Esta iniciativa de reemplazo de los motores de combustión por motores eléctricos impulsa el desarrollo y ayuda del sistema ecológico, debido a que existen las nuevas centrales de generación eléctrica como son las hídricas, eólicas y centrales fotovoltaicas que permitirán el abastecimiento de dichas unidades.

Para solventar la capacidad de energía de dichas embarcaciones se necesita un costo más bajo que el de uso de combustibles fósiles a mas que se podría implementar de manera puntual una central fotovoltaica para el abastecimiento en lugares recónditos como se tiene en zonas protegidas permitiendo que la inversión final del costo de uso de las embarcaciones ligeras para transporte fluvial sea más óptimo.

El peso de la estructura de soporte de los motores eléctricos con respecto a los de combustión fuera de borda permite utilizar materiales compuestos, aligerando el peso de la embarcación y permitiendo un mayor desempeño en la autonomía de navegación.

Los componentes eléctricos, electrónicos y alimentación energética (batería) cuentan con protección ip67 para daños por corrosión y humidificación, permitiendo una mayor durabilidad y menor gasto en costos de mantenimiento en general con respecto a un sistema de accionamiento por motor fuera de borda.

Se consideraría una mayor profundización de dicha investigación para poder generar una mejor resolución de datos para poder obtener un modelo más específico y optimo dependiendo las diferentes zonas de vigilancia y transporte fluvial; debido a que este sistema puede adaptar un sistema de auto recarga mediante condiciones de celdas fotovoltaicas y de flujo de aire, mejorando así el tiempo de carga y la durabilidad de la embarcación.

\section{References}

[1] Salas M, Luco R, Ekdahl H. Energía solar para propulsión de embarcaciones fluviales Iquitos. International Conference IDS 2013.

[2] Volker B. Practical Ship Hydrodynamics. Butterworth Heinemann; 2011. 
[3] Barragán C. Estudio del impacto ambiental del tráfico marítimo Barcelona- Baleares. Barcelona; 2014

[4] Muñoz JM. Las infraestructuras portuarias en Andalucía: Balance del periodo 1996 autonómico. Revista de Estudios Regionales. n 44:241-256.

[5] Aldeanueva F. Distribución y circulación de masas de agua en el golfo de Cádiz: variabilidad inducida por el forzamiento meteorológico [Tesis Doctoral]. Universidad de Málaga.

[6] Pastor H, Garcia J, Casafon M. Embarcaciones solares, una evolución al transporte marino en las islas Galápagos Ecuador. ISEREE - INER 2013.

[7] Salas M, Luco R, Ekdahl H. Energía solar para propulsión de embarcaciones fluviales Iquitos. International Conference IDS 2013.

[8] Volker B. Practical ship hydrodynamics. Butterworth Heinemann; 2011.

[9] Barragán C. Estudio del impacto ambiental del tráfico marítimo Barcelona- Baleares. Barcelona; 2014.

[10] Muñoz JM. Las infraestructuras portuarias en Andalucía: balance del periodo 1996 autonómico. Revista de Estudios Regionales. 44:241-256.

[11] Aldeanueva F. Distribución y circulación de masas de agua en el golfo de Cádiz: variabilidad inducida por el forzamiento meteorológico [Tesis Doctoral]. Universidad de Málaga.

[12] Pastor H, Garcia J, Casafon M. Embarcaciones solares, una evolución al transporte marino en las islas Galápagos Ecuador. ISEREE - INER 2013.

[13] Salas M, Luco R, Ekdahl H. Energía solar para propulsión de embarcaciones fluviales Iquitos. International Conference IDS 2013

[14] Volker B. Practical Ship Hydrodynamics. Butterworth Heinemann; 2011.

[15] Barragán C. Estudio del impacto ambiental del tráfico marítimo Barcelona- Baleares. Barcelona; 2014.

[16] Muñoz JM. Las infraestructuras portuarias en Andalucía: Balance del periodo 1996 autonómico. Revista de Estudios Regionales. 44;241-256.

[17] Aldeanueva F. Distribución y circulación de masas de agua en el golfo de Cádiz: variabilidad inducida por el forzamiento meteorológico [Tesis Doctoral]. Universidad de Málaga.

[18] Pastor H, Garcia J, Casafon M. Embarcaciones solares, una evolución al transporte marino en las islas Galápagos Ecuador. ISEREE - INER 2013

[19] Salas M, Luco R, Ekdahl H. Energía solar para propulsión de embarcaciones fluviales Iquitos. International Conference IDS 2013

[20] Volker B. Practical ship hydrodynamics. Butterworth Heinemann; 2011.

[21] Barragán C. Estudio del impacto ambiental del tráfico marítimo Barcelona- Baleares. Barcelona; 2014. 
[22] Muñoz JM. Las infraestructuras portuarias en Andalucía: Balance del periodo 1996 autonómico. Revista de Estudios Regionales. 44;241-256.

[23] Aldeanueva F. Distribución y circulación de masas de agua en el golfo de Cádiz: variabilidad inducida por el forzamiento meteorológico [Tesis Doctoral]. Universidad de Málaga.

[24] Pastor H, Garcia J, Casafon M. Embarcaciones solares, una evolución al transporte marino en las islas Galápagos Ecuador. ISEREE - INER 2013 